\title{
Article \\ Effects of Resonant Electromagnetic Fields on Biofilm Formation in Pseudomonas aeruginosa
}

\author{
Janus A. J. Haagensen ${ }^{1}$, Michael Bache ${ }^{2}$, Livio Giuliani ${ }^{3}$ and Nikolaj S. Blom ${ }^{2,4, *(D)}$ \\ 1 Novo Nordisk Foundation Center for Biosustainability, Technical University of Denmark (DTU), Building 220, \\ Kemitorvet, 2800 Kongens Lyngby, Denmark; jajh@dtu.dk \\ 2 Department of Chemical Engineering, Technical University of Denmark (DTU), Soltofts Plads 228A, \\ 2800 Kongens Lyngby, Denmark; mbac@dtu.dk \\ 3 Steering Committee, International Commission for Electromagnetic Safety (ICEMS), Viale Venezia 7, \\ 30171 Venice, Italy; giuliani.livio@gmail.com \\ 4 Department of Biotechnology and Biomedicine, Technical University of Denmark (DTU), Soltofts Plads 221, \\ 2800 Kongens Lyngby, Denmark \\ * Correspondence: nblom@dtu.dk
}

check for updates

Citation: Haagensen, J.A.J.; Bache, M.; Giuliani, L.; Blom, N.S. Effects of Resonant Electromagnetic Fields on Biofilm Formation in Pseudomonas aeruginosa. Appl. Sci. 2021, 11, 7760. https://doi.org/10.3390/app11167760

Academic Editor: Anthony William Coleman

Received: 5 July 2021

Accepted: 20 August 2021

Published: 23 August 2021

Publisher's Note: MDPI stays neutral with regard to jurisdictional claims in published maps and institutional affiliations.

Copyright: (c) 2021 by the authors. Licensee MDPI, Basel, Switzerland. This article is an open access article distributed under the terms and conditions of the Creative Commons Attribution (CC BY) license (https:// creativecommons.org/licenses/by/ $4.0 /)$.
Featured Application: Weak resonant magnetic fields may provide a non-chemical application towards combating antimicrobial resistance such as biofilm formation by pathogenic bacteria.

Abstract: The global rise of antimicrobial resistance (AMR) constitutes a future health threat and dictates a need to explore alternative and non-chemical approaches. The aim of this study was to explore the use of weak resonant electromagnetic fields as a method to disrupt biofilm formation of a pathogenic bacterium in cystic fibrosis patients. We developed a bioresonance laboratory setup able to distinguish between changes in planktonic growth and changes in biofilm formation and showed that certain resonant frequencies were able to affect biofilm formation without affecting planktonic growth. In addition, we show that the ambient day-to-day magnetic field affects biofilm formation in a non-consistent manner. Overall, we conclude that our assay is suitable for studying the potential of resonant magnetic fields as a treatment and prevention strategy to prevent biofilm infections, and that certain resonant frequencies may be used as future medical applications to combat antimicrobial resistance.

Keywords: bioresonance; antimicrobial resistance; microbial biofilm; infection; ion cyclotron resonance

\section{Introduction}

Widespread use of antibiotics has led to an increase in antimicrobial resistance (AMR), exemplified by the pathogenic bacteria Methicillin-resistant Staphylococcus aureus (MRSA) and Pseudomonas aeruginosa, the latter being a major challenge for cystic fibrosis patients [1]. Today, this is a threat to people with compromised immune systems, and tomorrow it may be a threat to all of humanity [2]. Consequently, there is an urgent need and a race against time to discover and invent new compounds or methods of combating life threatening infectious diseases, not only with regards to bacterial pathogens, but also viral pathogens, as currently witnessed (anno 2020) by the global COVID-19 pandemic.

Quantum physics has shown that the basic constituents of nature exist both as particles and waves. Medicine and biology have been highly successful in treating life from a particle perspective, which might explain why the wave aspect of biology was largely neglected and considered insignificant, even controversial. This may be due to a difficulty in replicating experiments and the belief that non-thermal radiation does not affect living systems. However, it is well known that weak forces, resonating at the right frequency, may have strong effects, as witnessed by opera singers' voices shattering crystal glasses and stable winds collapsing suspension bridges. Furthermore, rhythms play a fundamental role in living systems and rhythms are a musical expression of the modulation of frequencies [3]. 
Experiments now demonstrate that low-energy, highly specific resonant electromagnetic fields (EMF) affect living systems, e.g., the induction of stem cell differentiation or interference of bacterial growth [4,5]. A medical therapeutic breakthrough occurred in 2011, when the first resonant field-based therapy for treating brain tumors in terminal patients was approved for clinical use by FDA (reviewed in [6]).

\section{The Relationship between Resonant Electromagnetic Fields, Coherence in Water, and Biological Effects}

The mechanistic understanding of these phenomena has been lacking behind the observable effects; however, the pieces are starting to come together.

A breakthrough was obtained in the 1980-1990s, when scientists in USA and Russia independently showed that weak, extremely low frequency (ELF) magnetic fields at specific frequencies, tuned with the ratio of charge and mass of molecules dissolved in aqueous solution, are able to induce an electric current peak within an electrolytic cell $[7,8]$. These molecules included biologically relevant ions, amino acids, and nucleic acids, thus establishing the link between the ion cyclotron resonance (ICR) and living systems described by Equation (1): the ICR frequency, $\omega_{\mathcal{c}}$, is expressed as function of the ion charge-to-mass $(z / m)$ ratio and the strength of the static magnetic field B:

Equation (1):

$$
\omega_{C}=\frac{z B}{2 \pi m}
$$

The claim and observations that an induced Lorentz force many orders of magnitude lower than the Brownian force should have an effect on specific ions was questioned for a long period [9], until new theories of water came to the forefront in the frame of Quantum Electrodynamics (QED). This theory claims that water is a biphasic liquid, containing a phase of coherent, self-organized clusters of molecules, capable of storing additional energy, analogous to the coherent form of light known as laser [10]. The coherent clusters or domains flicker in and out of existence in the order of tenth of picoseconds. Only clusters stabilized by surfaces have lifetimes long enough to store and release energy from the ambience [11,12]. Experimental evidence of this theory has been supported by experimental findings using various spectroscopic methods, including infrared and near-infrared spectroscopy and X-ray studies of supercooled water [13-16].

As quoted above [9], Adair R.K. formulated the claim early that a weak electromagnetic field in the ELF band $(\leq 60 \mathrm{~Hz})$ induces a Lorentz force in water, which is several orders of magnitude lower than the Brownian force at room temperature. If we consider that the cytoplasm is a bath, where several species of molecules are dissolved in an aqueous solution, we should thus conclude that no chemical reaction can happen in cytoplasm due to a weak electromagnetic field. However, the theory of Adair R.K. was formulated within the framework of classical physics and before QED was fully developed to describe coherence in the matter [17]. There was also the false belief that quantum physics could not apply to the macroscopic phenomenon of living matter, despite the early warning of Erwin Schrödinger [18]. In 2002, the first explanation [19] of the quantum mechanism underlying the effect, earlier described by Liboff A.R. [7], was published. It describes the arising of ion currents in water under the ion cyclotron resonance (ICR) due to weak electromagnetic fields in the ELF band in the presence of a static magnetic field (in the order of the geomagnetic one), and of a static electric field in the order of the one existing across a eukaryotic cell membrane (app. $-100 \mathrm{mV}$ ).

However, the crucial point was to extend the mechanism to include biomolecules, i.e., bioactive ions or zwitterions (dipoles) in prokaryotic or eukaryotic cells. In 2006, Zhadin et al. demonstrated how a weak electromagnetic signal in the ELF band is indeed able to trigger the release of a bioactive molecules and ion currents [20].

The mechanism of the Zhadin effect can be summarized in three steps: (1) first, zwitterions and ions are captured by coherent clusters and are located on the boundary, provided their absorption peaks are close to the ones of water (mainly to the peaks corresponding 
to the electron excitation or to the phonons vibration); (2) within the cluster, zwitterions are ionized due to the action of the high electromagnetic vector potential gradient, at the boundary of the water cluster [21]; and (3) under the push of a proper ICR magnetic field, ions escape, provided by the energy of the cluster, and are accelerated by an electric field, provided by the transmembrane electric gradient. In addition, the Zhadin effect is based on the increase of the conductivity of the aqueous solution, the so-called protonation of water, as we detail in the discussion section $[5,22]$.

All this presumed, we hypothesized that prokaryotic cells as bacteria should also be subjected to the electromagnetic interaction provided by ion cyclotron resonance, and we argued that ICR should interfere differently on the prokaryotic cells of bacteria and upon the secreted biofilm due to the different content of water.

Indeed, pioneer studies have shown that exposure to electric and electromagnetic fields affect the growth of planktonic and biofilm forming bacteria and may enhance the efficacy of antibiotics [23,24]. This includes the bacterial pathogen Pseudomonas aeruginosa, which colonizes the lungs of patients with cystic fibrosis and reduces their lung capacity. In this exploratory pilot study, we have chosen P. aeruginosa, and investigate if bacteria exposed to an oscillating, pulsed electromagnetic field of extremely low intensity and frequency are affected in their growth pattern and ability to adhere to surfaces and eventually form biofilm.

In most laboratory experiments, the control condition refers to a situation unaffected by variable conditions such as chemical and physical factors. However, the ambient field of the electromagnetic environment is rarely taken into consideration but may vary from day to day. Changes in solar or electrical storms, geomagnetic conditions, telecommunication equipment nearby, or the activity of large equipment (freezers, centrifuges, etc.) may influence the local and ambient electromagnetic environment. This challenge was addressed by using a mu-metal alloy amagnetic chamber shielding external low-frequency magnetic fields.

After pilot experiments, we found that the most stable results came when using the ICR frequency of the potassium ion, $\mathrm{K}^{+}$, at a certain static magnetic field strength (10 microT). The observation that targeting the biologically important $\mathrm{K}^{+}$ion can modify bacterial physiology was also supported by a previous study [24].

\section{Materials and Methods}

\subsection{Bioresonance Laboratory}

A bioresonance lab was established, consisting of a temperature-controlled $\left(37^{\circ} \mathrm{C}\right)$ room equipped with a shielded cylindrical exposure chamber made of $\mathrm{Mu}$-metal alloy (length $68 \mathrm{~cm}$, diameter $22 \mathrm{~cm}$; model ZG209, Magnetic Shield Corporation, Bensenville, IL, USA) (see Figure 1). Baseline magnetic field strength inside the shielded exposure chamber was reduced to less than 60 nanoTesla, almost a thousand-fold reduction from the ambient geomagnetic field of app. 40 microTesla. 


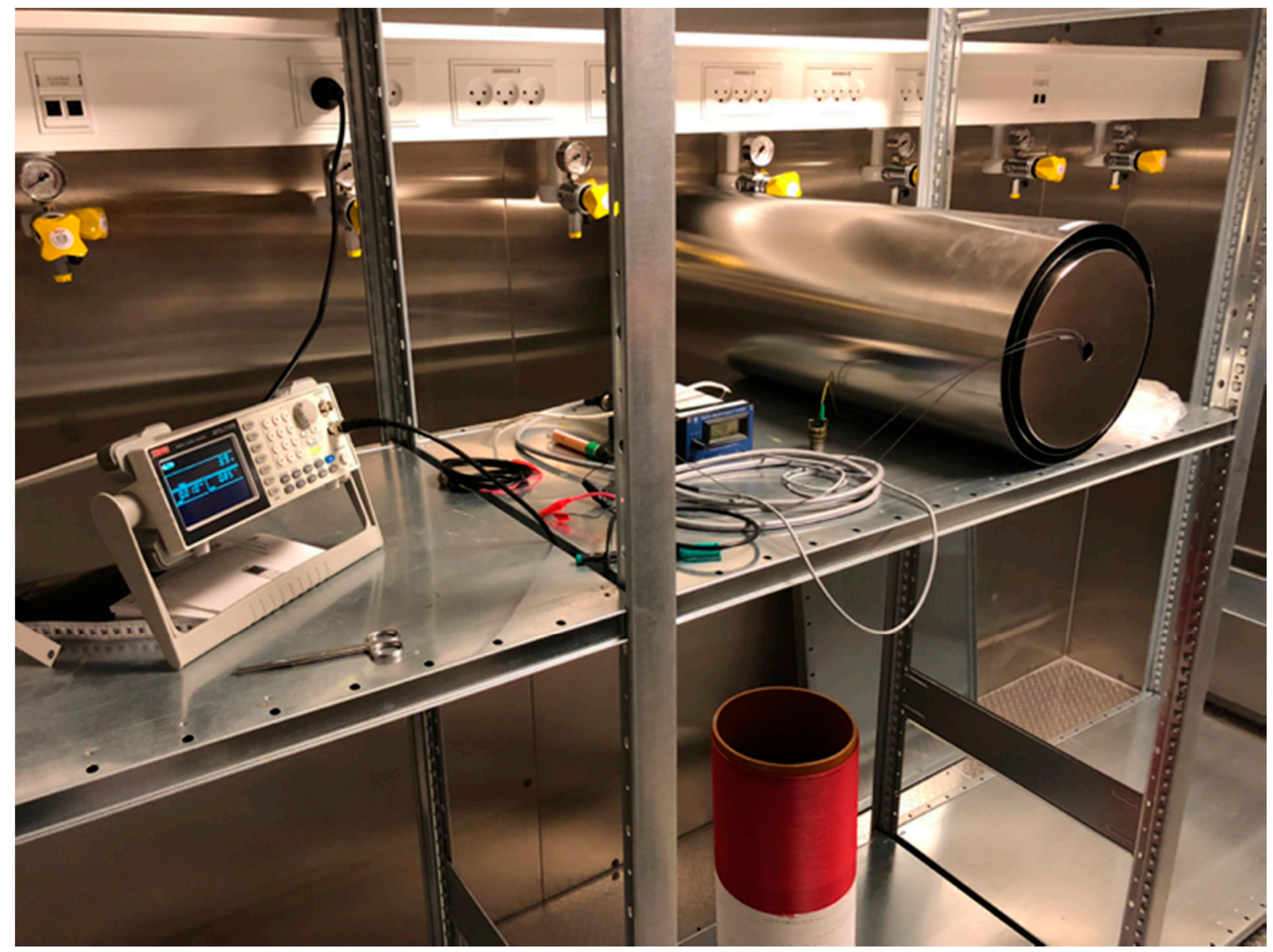

Figure 1. The bioresonance lab is temperature-controlled at $37^{\circ} \mathrm{C}$ and includes a function generator (left), a magnetometer (center), a triple-layer amagnetic mu-metal cylinder (right), and a custom-made solenoid (red/white, bottom) that fits inside the mu-metal cylinder.

\subsection{Exposure System and Procedure}

A custom-made Helmholtz coil/solenoid was made from a hard PVC tube (diameter of $20 \mathrm{~cm}$ and length $60 \mathrm{~cm}$ ) and windings of copper wire connected to a function generator. The generated fields were monitored with a custom-made magnetometer (accuracy: $+/-56 \mathrm{nT}$ ). Static magnetic fields in the range of 10-40 microTesla and alternating fields in the range of 100-300 nanoTesla were used when exposing bacteria. Frequencies of the alternating field were in the range of $3-13 \mathrm{~Hz}$.

Exposure was controlled by a function generator (model RS Pro 5 MHz AFG-21100) supplying a DC field of 10 to 30 microTesla (corresponding to 0.73 to 2.55 Volt DC) and an overlaid AC field of 130 to 300 nanoTesla (at 3.9 to $11.7 \mathrm{~Hz} / 0.130$ to $0.390 \mathrm{~V} \mathrm{AC}$ ).

The following exposure conditions were used in this study: (1) Exposure inside the amagnetic chamber by a specified DC field and a specified AC field of a given frequency ("exposed"); (2) Zero exposure or shielding inside the amagnetic chamber ("zero"); or (3) exposure to ambient field outside of chamber on the lab bench ("ambient").

Most exposures were carried out using a frequency of $3.9 \mathrm{~Hz}$, corresponding to the ICR of $\mathrm{K}^{+}$at a static magnetic field of $10 \mathrm{uT}$ according to Equation (1). The selection of the potassium cation $\mathrm{K}^{+}$as the primary target was based on findings observed by $\mathrm{Di}$ Bonaventura et al., which showed that the $\mathrm{K}^{+}$ICR had significant biological impact [24]. 


\subsection{Microbiological Growth and Biofilm Assay}

The experiments were performed using the biofilm-forming bacterial strain Pseudomonas aeruginosa, PAO1, and are based on the biofilm adhesion assay in 96-well peg-lid microtiter plates described previously [25].

\subsubsection{Inoculum Standardization}

Each assay was started from an overnight bacterial culture of P. aeruginosa (PAO1) grown in LB medium, diluted to OD600 $=0.05$, and 150 microliters aliquoted into 96-well peg-lid microtiter plates (Nunclon Delta Surface Cat. No.167008, Nunc TSP Cat. No.445497, Thermo Scientific, Waltham, MA, USA), as reported previously [26].

\subsubsection{Assay for Planktonic Bacterial Growth}

After $20 \mathrm{~h}$ of incubation, planktonic bacterial growth was monitored by measuring the OD600 for each well using a plate reader (Synergy H1, BioTek, Agilent, Santa Clara, CA, USA).

\subsubsection{Assay for Biofilm Adhesion to Polystyrene Pegs}

The ability of the PAO1 strains to adhere to and form biofilms on the polystyrene pegs was analyzed by crystal violet staining, as reported previously [27]. Briefly, bacteria were allowed to growth for $20 \mathrm{~h}$ in the peg-lid static system. Hereafter, the peg-lid was transferred to a "washing microtiter plate" with $180 \mu \mathrm{L}$ saline solution (PBS) to wash the pegs and remove non-adhering cells. The peg-lid was then transferred to a microtiter plate with $160 \mu \mathrm{L} 0.1 \%$ crystal violet (CV) and left to stain for $20 \mathrm{~min}$. To remove unbound crystal violet, the lids were then washed three times in three individual "washing microtiter plates" with $180 \mu \mathrm{L}$ PBS. Bacterial adhesion to the peg-lid was measured by detaching adhering CV-stained cells through placing the peg-lids in a microtiter plate containing $180 \mu \mathrm{L} 99 \%$ ethanol. A plate reader (Synergy H1, BioTek, Agilent, Santa Clara, CA, USA) was then used to measure the CV optical density at OD590.

\subsubsection{Calculation of the Relative Biofilm Adhesion Units}

Relative biofilm formation or adhesion units were calculated as the OD590/OD600 ratio for a given well in a given plate, as described previously [25]. Relative biofilm adhesion units for a given plate were normalized to the shielded ("zero") control and statistical analysis was performed using a t-test.

\section{Results}

3.1. Planktonic Bacterial Growth Does Not Vary Statistically under Various Magnetic Field Conditions

In a series of experiments, PAO1 cells in microtiter plates were exposed to either the ambient field ("ambient"), a shielded field ("zero"), or a specific EMF of $3.9 \mathrm{~Hz}$ ("exposed"), as described above. In some series, the ambient condition was omitted.

No difference in absolute growth rate was observed (data not shown). After $20 \mathrm{~h}$ of exposure, the planktonic bacterial growth was recorded as OD600 values and normalized to the value for the "shielded/zero" condition for that series (set at 100). For individual wells in the microtiter plate, grown under identical conditions, the maximal standard deviation was $14 \%$.

Figure 2 shows the normalized average planktonic growth values for each of the three experimental conditions. Ambient and exposed plates showed values of 98 and 105, respectively, when normalized to the shielded condition (value 100). The average of several experimental series for each condition showed a consistent standard deviation of $12 \%$ in all cases. 


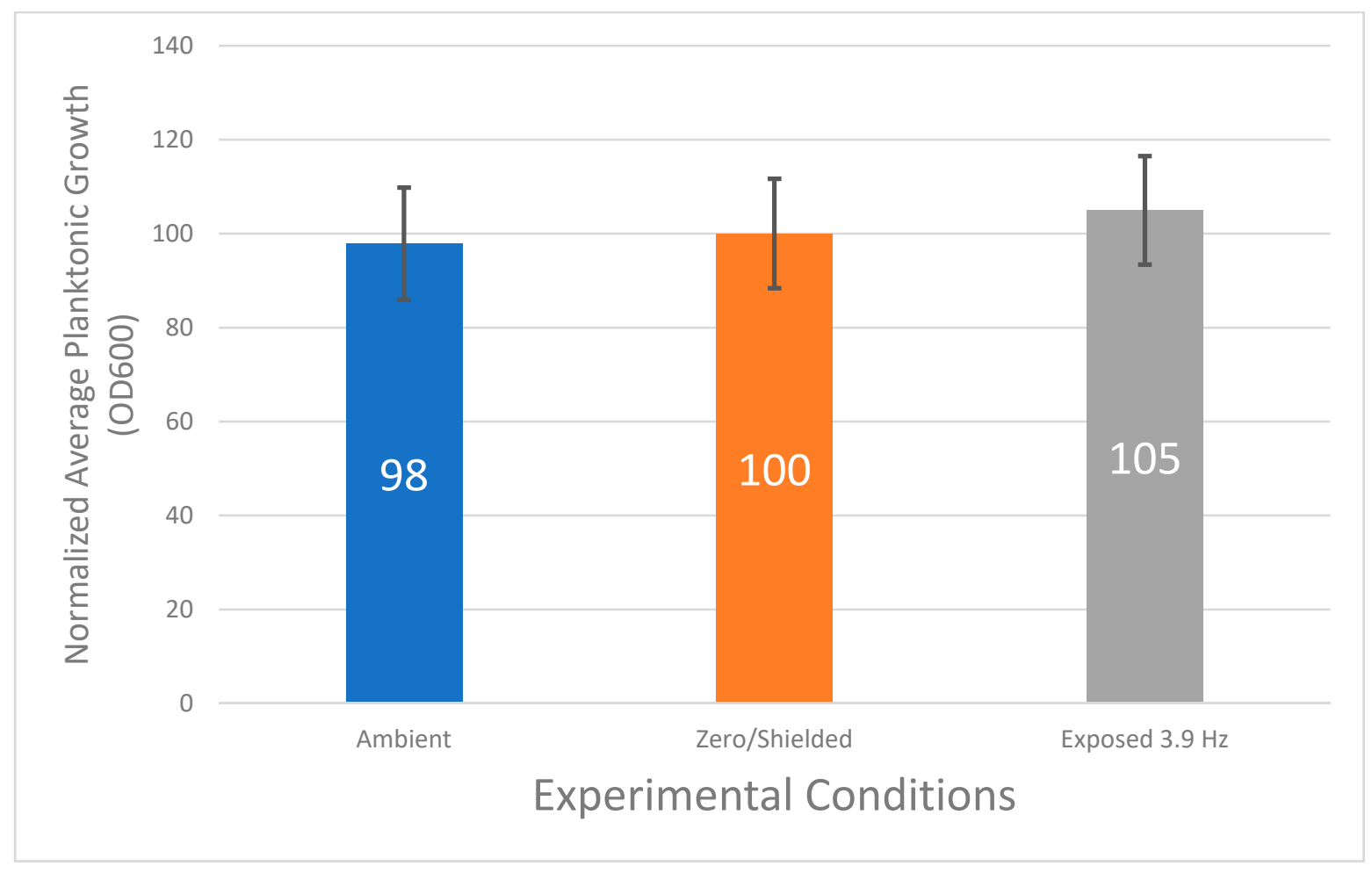

Figure 2. Effect on planktonic bacterial growth (measured as optical density at $600 \mathrm{~nm} / \mathrm{OD} 600$ ) for three different growth conditions, "Ambient/room", "Zero/shielded", or "Exposed/3.9 Hz". Y-axis show normalized OD600 values relative to the "Zero/shielded" condition (value 100) for each series. Average and error bars are for $n=2$ (Ambient), $n=5$ (Zero), or $n=5$ (Exposed) experimental series, respectively.

In conclusion, no statistically significant difference could be observed on planktonic bacterial growth between experimental conditions tested in this study.

\subsection{The Effect of the Ambient Electromagnetic Field on Bacterial Biofilm Formation Is Not Constant}

To test the effect of the ambient electromagnetic field on bacterial biofilm formation, PAO1 cells grown under ambient or shielded (hypomagnetic) conditions were investigated. Under shielded conditions, not even the influence of the static geomagnetic field (typically 30 to 50 microTesla) should be sensed by the bacteria. If ambient and shielded conditions are different but constant, we should expect to see a constant ratio of biofilm formation between the two conditions.

Figure 3 shows the relative biofilm formation from several series of experiments performed over a period of several months. The calculated relative biofilm formation at ambient conditions performed at different dates, ranging from 102\% (series A) to $133 \%$ (series P) of control ("Zero"). 


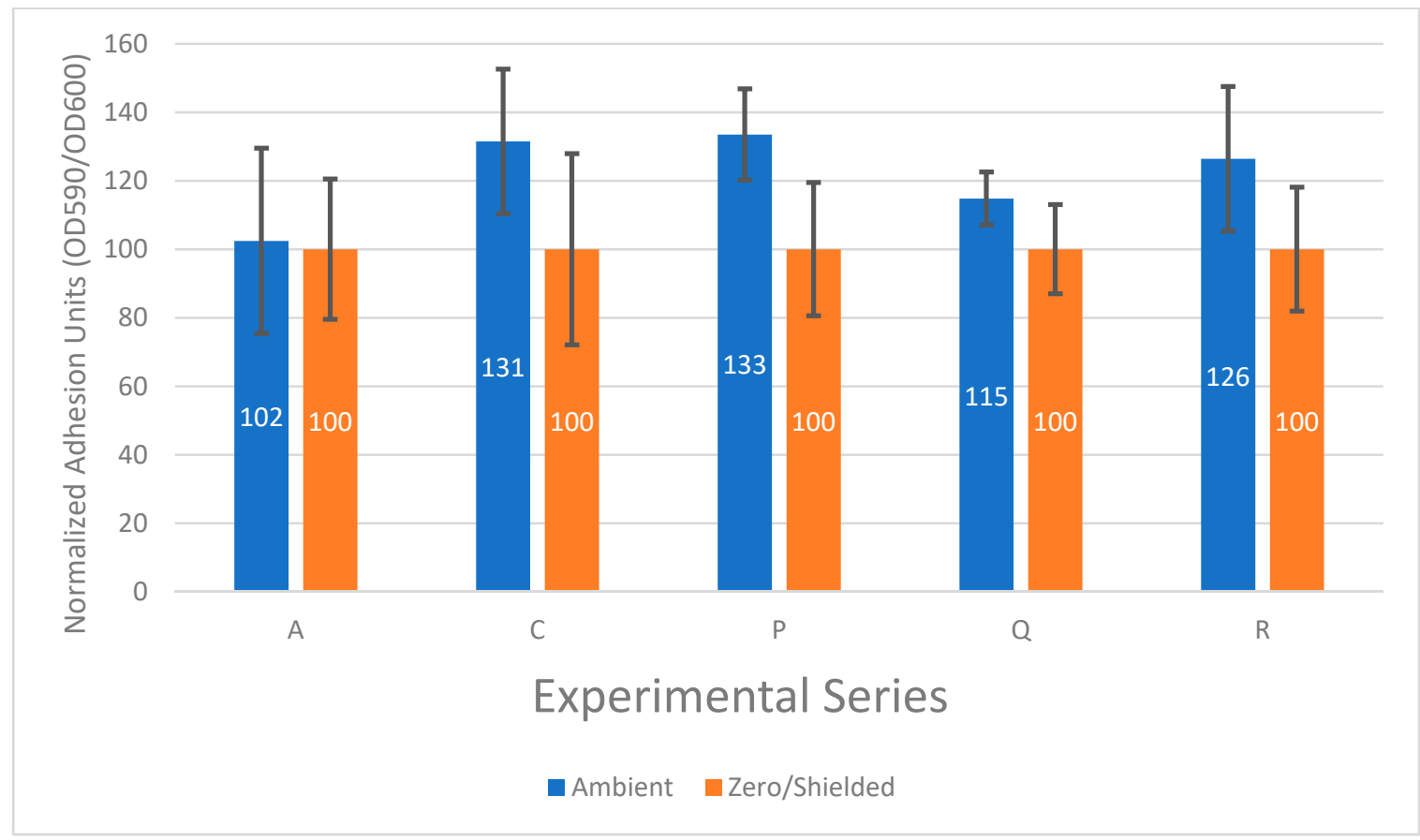

Figure 3. Effect of ambient versus shielded conditions on Biofilm Adhesion Units (ratios of OD590/OD600) and normalized to control condition "Zero/Shielded (value at 100)". Data points and standard deviations shown for five experimental series (A-R) under two conditions: "Ambient/room", "Zero/shielded". Y-axis show normalized biofilm ratios relative to the "Zero/shielded" condition (value 100).

In conclusion, the relative biofilm formation of PAO1 cells grown under ambient conditions did not appear constant relative to the shielded condition. Based on this finding, subsequent experiments used the cells grown under shielded (Zero) condition as the control.

\subsection{Biofilm Formation Is Consistently Inhibited at a Specific Frequency}

PAO1 bacteria in 96 well microtiter plates were exposed for $20 \mathrm{~h}$ on six different occasions (series) to an oscillating field of $3.9 \mathrm{~Hz}$ (130 nanoT AC overlayed on a 10 microT DC/static field). As a control, PAO1 bacteria in 96-well microtiter plates were left unexposed inside the shielded chamber for $20 \mathrm{~h}$ (labelled 'Zero').

Figure 4 illustrates that a reduction of bacterial biofilm formation was consistently observed when PAO1 cultures were exposed to an oscillating field of $3.9 \mathrm{~Hz}$. An average of $27 \%$ reduction was observed over six experimental series ranging from $16 \%$ to $41 \%$ reduction (see Figure 4). Statistical significance was calculated using a $t$-test. $p$-values ranged from $p<2 \times 10^{-8}$ to $p<5 \times 10^{-26}$, showing significant changes in bacterial biofilm formation after exposure to the oscillating field versus control. Since each microtiter plate consists of 96 technical replicates, the degrees of freedom are significant, and thus strengthen the calculated $p$-values. 


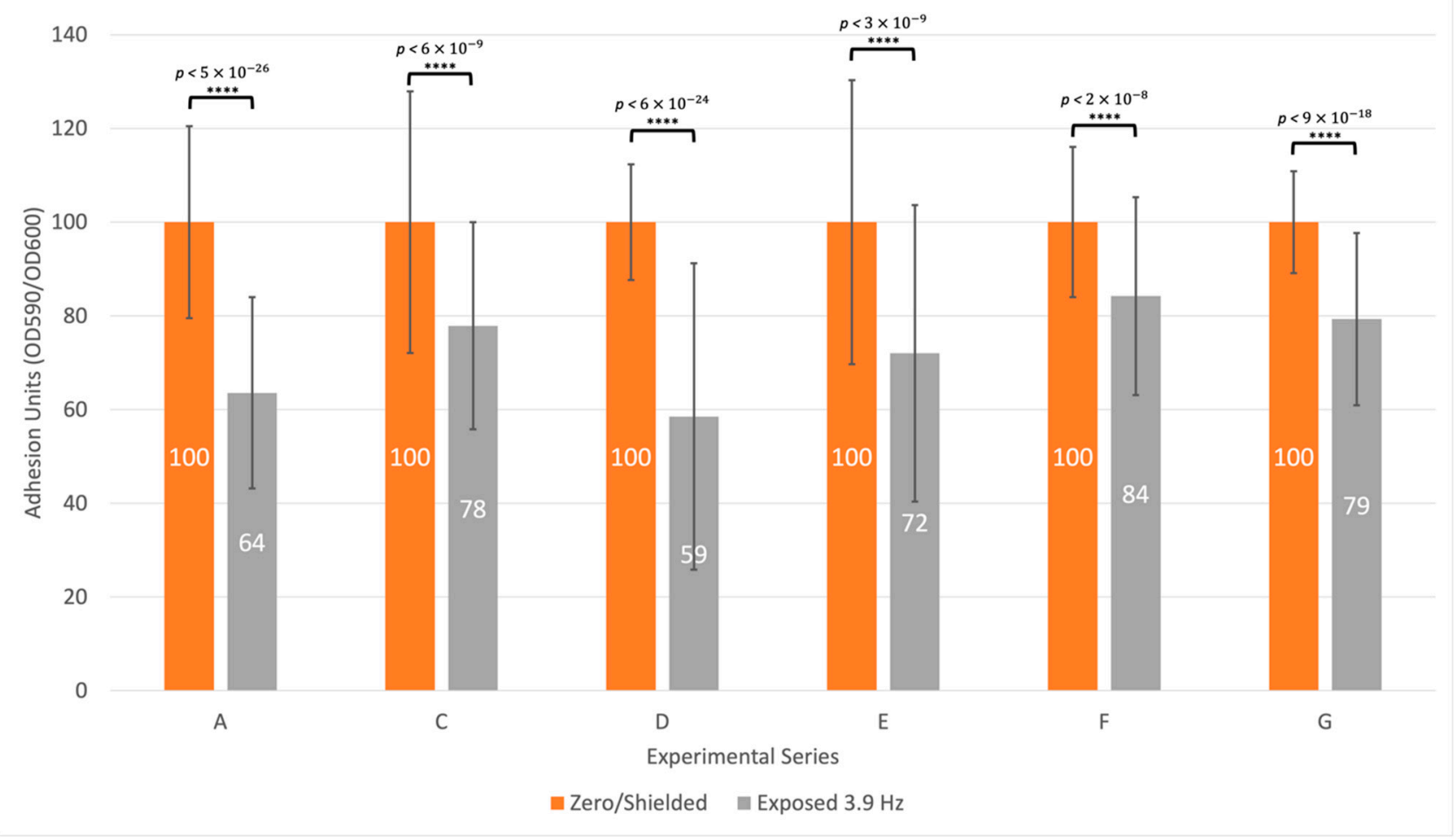

Figure 4. Effect of shielded vs. exposed conditions on biofilm adhesion units (ratios of OD590/OD600) normalized to control condition "Zero/Shielded". Data points from six experimental series (A-G) are shown at two different conditions: "Zero/shielded" and "Exposed/3.9 Hz". Y-axis show normalized values relative to the "Zero/shielded" condition (value 100). Standard deviation for each microtiter plate and statistical $p$-values (paired $t$-test) are indicated (**** signifies $p<0.0001$ ).

Since planktonic growth was not affected (see Figure 2), the exposure of PAO1 bacteria to a combination of a static (DC) magnetic field of 10 microTesla overlaid with a $3.9 \mathrm{~Hz}$ oscillating (AC) magnetic field of 130 nanoTesla may affect the ability of the tested cells of P. aeruginosa to adhere and thus develop infectious biofilms.

\subsection{Other Frequencies Tested Showed Variable and Inconclusive Results}

To further investigate the possibility that targeting the theoretical ICR of $\mathrm{K}^{+}$experiments were performed using a set of parameters three times larger, i.e., a static field of 30 microT $(3 \times 10)$ and a calculated ICR frequency of $11.7 \mathrm{~Hz}(3 \times 3.9 \mathrm{~Hz})$, according to Equation (1).

These experiments were inconclusive and highly variable (results not shown).

\section{Discussion}

The aim of this study was to establish a bioresonance laboratory and explore the use of weak resonant electromagnetic fields as a method to disrupt biofilm formation in vitro of a known pathogenic bacterium, Pseudomonas aeruginosa. P. aeruginosa is a pathogen that is dominant in patients suffering from cystic fibrosis and any new approach to inhibit the infectious biofilm formation of this bacterium in the lungs of CF patients would be highly important and have clinical relevance.

In summary, the following three results were obtained: (1) no effects of a shielded or specific magnetic field on the growth of planktonic, i.e., non-biofilm forming bacteria, (2) a variable or non-constant effect of the ambient or environmental electromagnetic field on bacterial biofilm formation, and (3) an inhibitory effect on bacterial biofilm formation at a specific frequency of the electromagnetic field. 
With regard to the first finding, it has been reported by others that electrical and magnetic fields may affect planktonic bacterial growth (see [28] and references therein). However, in the present study, no such effect was observed. It might be argued that OD600 does not necessarily correspond to the number of viable bacteria and could also include dead cells. However, a few live/dead stains and growth curves were performed nonsystematically, which indicated that there were no differences between the experimental conditions and that planktonic bacterial cultures were growing as expected.

The second finding related to the ambient electromagnetic field. When looking at the ability to form biofilm on the peg-lids, it was found that the ambient or environmental electromagnetic field in our research facility did have a non-constant effect on bacterial biofilm formation. Thus, the ambient electromagnetic field caused by natural and artificial phenomena, cannot be assumed to be constant from one experimental period to the other. For example, in any modern-day laboratory setting, electrical equipment such as refrigerators, telecommunication equipment, etc., may produce a non-negligible $50 \mathrm{~Hz}$ magnetic field of 500 nanoT or more. It is therefore important to control for this using, for example, a mu-metal cylinder shielding against the ambient magnetic fields.

Studying formation of bacterial biofilm as opposed to planktonic growth can be challenging, since biofilm can form on most surfaces that a bacterial culture touches. In the case of standard microtiter plates, the bottom, sides, and rim surfaces are subject to biofilm formation. This presented a great challenge to early investigations of microbial biofilm formation, since it was very difficult to separate the planktonic and attached fractions in a standard well or dish. In 1999, the peg-lid principle was introduced, which showed much improved reproducibility, due to the ability to easily separate a defined portion of the surface available (the pegs) to the bacteria [29]. Furthermore, the use of the 96-well microtiter format basically allows for performing 96 technical replicates in one batch, and thus improving the statistical power greatly.

The third finding in this study was based on using the proper control and a statistically robust biological peg-lid assay as described above. Using a magnetically shielded bacterial culture as control, it was found that exposing the bacteria to a specific electromagnetic field of 10 microT and $3.9 \mathrm{~Hz}$ consistently showed an inhibitory and statistically significant reduction of biofilm formation. A series of six experiments all showed an inhibitory effect, on average a $27 \%$ reduction of biofilm formation.

The specific frequency of $3.9 \mathrm{~Hz}$ was calculated as the Ion Cyclotron Resonance (ICR) of the potassium ion, $\mathrm{K}^{+}$, at a specific magnetic field strength. We found that tripling the magnetic field and tuning the ICR frequency did not show the same consistency as observed using the $3.9 \mathrm{~Hz}$ frequency. This would be expected if the resonance effect derives purely from a single ion species. However, as mentioned earlier, the effects observed are likely dependent on the protonation of water as well as the biologically relevant ion, in this case $\mathrm{K}^{+}$.

The term protonation of water here means the exposure of the aqueous solution to the ICR frequency of the Zündel Cation, $\mathrm{H}_{3} \mathrm{O}^{+} \cdot\left(\mathrm{H}_{2} \mathrm{O}\right)$. In previous studies on the polymorphism of water, the role of the Zündel Cation, one of the most abundant hydronium hydrates, has been suggested to provide the needed energy for activating other cations. It was observed that the release of Zündel Cations and other hydronium hydrates enhances the conductivity of water, reducing $\mathrm{pH}$ and modifying its refraction index, thereby enhancing the small signal of ICR, and allowing ion currents aroused by ICR to overcome the environmental thermal barrier, $k T$ (where $k$ is the Boltzmann's constant and $T$ the absolute temperature). It was also observed that water works as an amplifier in the ELF band when hydronium hydrates are able to enhance such polymorphisms [30,31]. Such effect of ICR on hydronium hydrates has been confirmed in the present experiment thanks to differential results depending on the presence of the Zündel Cation component in the $\mathrm{K}^{+}$-ICR that was administrated to bacteria, in the way described below.

As the static magnetic field (10 microT) consistently showed effects on bacterial biofilm, we expect an ICR of $\mathrm{K}^{+}$of $3.9 \mathrm{~Hz}$ and $4.1 \mathrm{~Hz}$ of the Zündel Cation, $\mathrm{H}_{3} \mathrm{O}^{+} \cdot\left(\mathrm{H}_{2} \mathrm{O}\right)$, thus a 
$0.2 \mathrm{~Hz}$ difference. Considering the Half Width at Half Maximum (HWHM), I / 2, may vary depending on many parameters characterizing the resonance, including the $Q$ factor known from radio wave reception theory. It has previously been suggested that the HWHM is in the order of $10^{-1} \mathrm{~Hz}$, thus providing a plausible explanation for activating both $\mathrm{K}^{+}$ and $\mathrm{H}_{3} \mathrm{O}^{+} \cdot\left(\mathrm{H}_{2} \mathrm{O}\right)$ when using the 10 microT static field [8]. However, when all factors were tripled, the difference in calculated ICR was increased to $0.6 \mathrm{~Hz}(3 \times 0.2 \mathrm{~Hz})$ which is in the order of $1 \mathrm{~Hz}$ and may reduce the effect of nearby frequencies and the reproducibility of the experiment.

Thus, the methodology described here may serve as a starting point for developing novel strategies against the rising global challenge of antimicrobial resistance and impact clinical challenges such as chronic infections in ulcers, catheters, and urinary tracts.

Future directions may include the synergistic effects of resonant magnetic fields and antimicrobial compounds, and assay conditions mimicking clinically relevant situations. These may include biofilm formation in flow chambers and behavior of bacteria infecting organoids (small artificial organs). Molecular and biochemical investigations using metabolomics, transcriptomics, and proteomics approaches may also elucidate the biochemical pathways perturbed by resonant magnetic fields.

\section{Conclusions}

Our findings suggest that certain resonant magnetic fields may interfere with microbial biofilm formation and therefore provide a non-chemical tool to combat the rising antimicrobial resistance. We also demonstrate that a dedicated bioresonance laboratory equipped to investigate controlled magnetic fields shielded against the ambient magnetic fields is feasible and needed for future experiments.

Author Contributions: Conceptualization, N.S.B. and L.G.; methodology, J.A.J.H.; investigation, J.A.J.H. and M.B.; writing—original draft preparation, N.S.B.; writing—review and editing, N.S.B., M.B., J.A.J.H. and L.G.; funding acquisition, N.S.B. All authors have read and agreed to the published version of the manuscript.

Funding: This research was funded by VILLUM FONDEN, grant numbers 17449 (Direwaves) and 23323 (WaterStruc).

Institutional Review Board Statement: Not applicable.

Informed Consent Statement: Not applicable.

Acknowledgments: The authors would like to thank Lars W Pedersen at DTU Space and Georgios Kontogeorgis at DTU Chemical Engineering for technical assistance and project guidance.

Conflicts of Interest: The authors declare no conflict of interest. The funders had no role in the design of the study; in the collection, analyses, or interpretation of data; in the writing of the manuscript, or in the decision to publish the results.

\section{References}

1. Prestinaci, F.; Pezzotti, P.; Pantosti, A. Antimicrobial Resistance: A Global Multifaceted Phenomenon. Pathog. Glob. Health 2015, 109, 309-318. [CrossRef] [PubMed]

2. Toner, E.; Adalja, A.; Gronvall, G.K.; Cicero, A.; Inglesby, T.V. Antimicrobial Resistance Is a Global Health Emergency. Health Secur. 2015, 13, 153-155. [CrossRef] [PubMed]

3. Muehsam, D.; Ventura, C. Life Rhythm as a Symphony of Oscillatory Patterns: Electromagnetic Energy and Sound Vibration Modulates Gene Expression for Biological Signaling and Healing. Glob. Adv. Health Med. 2014, 3, 40-55. [CrossRef] [PubMed]

4. Lisi, A.; Ledda, M.; de Carlo, F.; Pozzi, D.; Messina, E.; Gaetani, R.; Chimenti, I.; Barile, L.; Giacomello, A.; D’Emilia, E.; et al. Ion Cyclotron Resonance as a Tool in Regenerative Medicine. Electromagn. Biol. Med. 2008, 27, 127-133. [CrossRef]

5. Giuliani, L.; D'Emilia, E.; Grimaldi, S.; Lisi, A.; Bobkova, N.; Zhadin, M.N. Investigating the Icr Effect in a Zhadin's Cell. Int. J. Biomed. Sci. 2009, 5, 181-186. [PubMed]

6. Fabian, D.; Guillermo Prieto Eibl, M.D.P.; Alnahhas, I.; Sebastian, N.; Giglio, P.; Puduvalli, V.; Gonzalez, J.; Palmer, J.D. Treatment of Glioblastoma (GBM) with the Addition of Tumor-Treating Fields (TTF): A Review. Cancers 2019, 11, 174. [CrossRef]

7. Liboff, A.R. Cyclotron Resonance in Membrane Transport. In Interactions between Electromagnetic Fields and Cells; Chiabrera, A., Nicolini, C., Schwan, H.P., Eds.; Plenum Press: New York, NY, USA, 1985; pp. 281-290. 
8. Zhadin, M.N.; Novikov, V.V.; Barnes, F.S.; Pergola, N.F. Combined Action of Static and Alternating Magnetic Fields on Ionic Current in Aqueous Glutamic Acid Solution. Bioelectromagnetics 1998, 19, 41-45. [CrossRef]

9. Adair, R.K. Constraints on Biological Effects of Weak Extremely-Low-Frequency Electromagnetic Fields. Phys. Rev. A 1991, 43, 1039-1048. [CrossRef]

10. Del Giudice, E.; Preparata, G.; Vitiello, G. Water as a Free Electric Dipole Laser. Phys. Rev. Lett. 1988, 61, 1085-1088. [CrossRef]

11. Arani, R.; Bono, I.; Giudice, E.D.; Preparata, G. Qed Coherence and the Thermodynamics of Water. Int. J. Mod. Phys. B 1995, 9 , 1813-1841. [CrossRef]

12. Comisso, N.; Giudice, E.D.; Ninno, A.D.; Fleischmann, M.; Giuliani, L.; Mengoli, G.; Merlo, F.; Talpo, G. Dynamics of the Ion Cyclotron Resonance Effect on Amino Acids Adsorbed at the Interfaces. Bioelectromagnetics 2006, 27, 16-25. [CrossRef]

13. Huang, C.; Wikfeldt, K.T.; Tokushima, T.; Nordlund, D.; Harada, Y.; Bergmann, U.; Niebuhr, M.; Weiss, T.M.; Horikawa, Y.; Leetmaa, M.; et al. The Inhomogeneous Structure of Water at Ambient Conditions. Proc. Natl. Acad. Sci. USA 2009, 106, 15214-15218. [CrossRef]

14. Taschin, A.; Bartolini, P.; Eramo, R.; Righini, R.; Torre, R. Evidence of Two Distinct Local Structures of Water from Ambient to Supercooled Conditions. Nat. Commun. 2013, 4, 2401. [CrossRef] [PubMed]

15. Renati, P.; Kovacs, Z.; De Ninno, A.; Tsenkova, R. Temperature Dependence Analysis of the NIR Spectra of Liquid Water Confirms the Existence of Two Phases, One of Which Is in a Coherent State. J. Mol. Liq. 2019, 292, 111449. [CrossRef]

16. Kim, K.H.; Späh, A.; Pathak, H.; Perakis, F.; Mariedahl, D.; Amann-Winkel, K.; Sellberg, J.A.; Lee, J.H.; Kim, S.; Park, J.; et al. Maxima in the Thermodynamic Response and Correlation Functions of Deeply Supercooled Water. Science 2017, 358, 1589-1593. [CrossRef] [PubMed]

17. Preparata, G. QED Coherence in Matter; World Scientific: Singapore, 1995; ISBN 978-981-02-2249-9.

18. Schrödinger, E. What Is Life? With Mind and Matter and Autobiographical Sketches; Canto; Cambridge University Press: Cambridge, UK, 1992.

19. Del Giudice, E.; Fleischmann, M.; Preparata, G.; Talpo, G. On the “Unreasonable” Effects of ELF Magnetic Fields upon a System of Ions. Bioelectromagnetics 2002, 23, 522-530. [CrossRef]

20. Zhadin, M.; Giuliani, L. Some Problems in Modern Bioelectromagnetics. Electromagn. Biol. Med. 2006, 25, 227-243. [CrossRef]

21. De Ninno, A.; Congiu Castellano, A. Deprotonation of Glutamic Acid Induced by Weak Magnetic Field: An FTIR-ATR Study. Bioelectromagnetics 2011, 32, 218-225. [CrossRef]

22. Giuliani, L.; Grimaldi, S.; Lisi, A.; D’Emilia, E.; Bobkova, N.; Zhadin, M. Action of Combined Magnetic Fields on Aqueous Solution of Glutamic Acid: The Further Development of Investigations. Biomagn. Res. Technol. 2008, 6, 1. [CrossRef]

23. Browne, M.; Foley, S.; Babck, N.; Olshefsky, S.; Holland, A.; Ahern, H. Non-Thermal Inhibition of Antibiotic Resistant Bacteria by Oscillating Pulsed Electric Fields (OPEF); The American Society of Microbiology: Denver, CO, USA, 2013.

24. Di Bonaventura, G.; Pompilio, A.; Crocetta, V.; De Nicola, S.; Barbaro, F.; Giuliani, L.; D’Emilia, E.; Fiscarelli, E.; Bellomo, R.G.; Saggini, R. Exposure to Extremely Low-Frequency Magnetic Field Affects Biofilm Formation by Cystic Fibrosis Pathogens. Future Microbiol. 2014, 9, 1303-1317. [CrossRef] [PubMed]

25. Falcone, M.; Ferrara, S.; Rossi, E.; Johansen, H.K.; Molin, S.; Bertoni, G. The Small RNA ErsA of Pseudomonas Aeruginosa Contributes to Biofilm Development and Motility through Post-Transcriptional Modulation of AmrZ. Front. Microbiol. 2018,9 , 238. [CrossRef]

26. Harrison, J.J.; Stremick, C.A.; Turner, R.J.; Allan, N.D.; Olson, M.E.; Ceri, H. Microtiter Susceptibility Testing of Microbes Growing on Peg Lids: A Miniaturized Biofilm Model for High-Throughput Screening. Nat. Protoc. 2010, 5, 1236-1254. [CrossRef]

27. Bartell, J.A.; Cameron, D.R.; Mojsoska, B.; Haagensen, J.A.J.; Pressler, T.; Sommer, L.M.; Lewis, K.; Molin, S.; Johansen, H.K. Bacterial Persisters in Long-Term Infection: Emergence and Fitness in a Complex Host Environment. PLoS Pathog. 2020, 16, e1009112. [CrossRef] [PubMed]

28. Freebairn, D.; Linton, D.; Harkin-Jones, E.; Jones, D.S.; Gilmore, B.F.; Gorman, S.P. Electrical Methods of Controlling Bacterial Adhesion and Biofilm on Device Surfaces. Expert Rev. Med. Devices 2013, 10, 85-103. [CrossRef] [PubMed]

29. Ceri, H.; Olson, M.E.; Stremick, C.; Read, R.R.; Morck, D.; Buret, A. The Calgary Biofilm Device: New Technology for Rapid Determination of Antibiotic Susceptibilities of Bacterial Biofilms. J. Clin. Microbiol. 1999, 37, 1771-1776. [CrossRef]

30. D’Emilia, E.; Giuliani, L.; Lisi, A.; Ledda, M.; Grimaldi, S.; Montagnier, L.; Liboff, A.R. Lorentz Force in Water: Evidence That Hydronium Cyclotron Resonance Enhances Polymorphism. Electromagn. Biol. Med. 2015, 34, 370-375. [CrossRef]

31. D’Emilia, E.; Ledda, M.; Foletti, A.; Lisi, A.; Giuliani, L.; Grimaldi, S.; Liboff, A.R. Weak-Field H3O+ Ion Cyclotron Resonance Alters Water Refractive Index. Electromagn. Biol. Med. 2017, 36, 55-62. [CrossRef] 